v. 01, n. 02 : pp. $18-32,2005$

ISSN $1808-0448$

D.O.I.: $10.3895 /$ S $1808-04482005000200002$
Revista Gestão Industrial

\title{
REQUISITOS DE IMPLANTAÇÃO DO QFD E RECOMENDAÇÕES PARA REDUZIR DIFICULDADES DE USO: Análise da Literatura Utilizando o Método AHP
}

\author{
REQUIREMENTS FOR QFD IMPLEMENTATION AND \\ RECOMMENDATIONS TO REDUCE DIFFICULTIES OF USAGE: A \\ LITERATURE ANALYSIS USING AHP
}

\author{
José Antonio Carnevalli ${ }^{1}$; Paulo Augusto Cauchick Miguel ${ }^{2}$; Felipe Araújo Calarge ${ }^{3}$ \\ ${ }^{1}$ UNIMEP; jacarneval@unimep.br \\ ${ }^{2}$ UNIMEP/USP; pamiguel@unimep.br \\ ${ }^{3}$ UNIMEP; fcalarge@unimep.br
}

Recebido para publicação em: 25/01/2005

Aceito para publicação em: 11/06/2005

\section{RESUMO}

O QFD é um importante método para o desenvolvimento de produtos. Este método traduz e identifica os principais requisitos dos clientes em especificações de projeto, desdobrando estas especificações para cada etapa do processo de desenvolvimento de um produção ou serviço. Entretanto, a literatura destaca várias dificuldades enfrentadas pelos usuários do método na implantação do QFD. Buscando inicialmente entender estas dificuldades está sendo realizada uma pesquisa bibliográfica que busca identificar as dificuldades, recomendações e pré-requisitos do uso do QFD. Especificamente neste artigo é apresentado o uso do AHP (Analytic Hierarchy Process), visando auxiliar na identificação das principais dificuldades, requisitos e recomendações do QFD. Futuramente esta análise será importante nas demais etapas desta pesquisa que busca estudar meios de minimizar algumas destas dificuldades.

Palavras-chave: QFD; AHP; Dificuldades no uso do QFD.

\section{Introdução}

Com o objetivo de ajudar as empresas a definirem os pontos críticos de controles de garantia da qualidade antes do início da produção, Akao iniciou estudos em 1966 adaptando uma tabela de pontos de controle na produção desenvolvida por Oshiumi (AKAO \& MAZUR, 2003). Com a integração da pesquisa desenvolvida por Akao com os estudos de Mizuno, Furukawa e Ishihara foi concluído o método do QFD (Quality Function Deployment - Desdobramento da Função Qualidade) da forma como hoje é conhecido (AKAO \& MAZUR, 2003; AKAO 1996). Atualmente, tal método encontra-se em utilização em diversos países do mundo, tais como África 
do Sul, Alemanha, Austrália, Brasil, Espanha, Estados Unidos, Itália, Japão, México, Reino Unido, Suécia dentre outros e não somente no desenvolvimento de produto mas, também no desenvolvimento de processos de fabricação, softwares, serviços, etc. (AKAO \& MAZUR, 2003; CHAN \& WU, 2002).

Apesar de ser utilizado em vários países, a aplicação do QFD não é fácil, e isso vem limitando o seu uso. No entanto, as empresas têm encontrado dificuldades no seu uso. Dentre essas dificuldades podem ser citadas (BOUCHEREAU \& ROWLANDS, 2000; CARNEVALLI, 2002; MARTINS \& ASPINWALL, 2001; MIGUEL, 2003; PARKIN et al. 2002): falta de comprometimento da equipe do QFD; falta de experiência no uso do método e dificuldades em se trabalhar com matrizes muito grandes; longo tempo para definir a qualidade projetada e montar as matrizes do QFD; análise de dados subjetivos; dentre outras.

Devido a estas razões, torna-se importante a realização de estudos que reduzam as dificuldades e incentivem o seu uso. Este é o objetivo da presente pesquisa: desenvolver um modelo sistêmico de implantação do QFD que elimine ou reduza algumas destas dificuldades, utilizando para esta finalidade o método AD (Axiomatic Design - Projeto Axiomático); ver Suh (1990). Atualmente, esta pesquisa está na fase de revisão e análise bibliográfica, sendo que foi concluída parte desta revisão. Neste artigo é apresentada a pesquisa bibliográfica que investiga dificuldades, recomendações e pré-requisitos do QFD (parte já apresentada em Carnevalli et al. 2004a). Os dados desta pesquisa foram organizados utilizando-se uma adaptação do diagrama de afinidades (método KJ de agrupamento). Para facilitar a análise destes dados, estes foram priorizados usando o método AHP (Analytic Hierarchy Process). Este artigo apresenta os métodos e técnicas de pesquisa adotados neste trabalho, a análise dos dados da literatura, bem como os resultados parciais obtidos até o momento.

\section{Exemplo de pesquisas para ajudar no uso do QFD}

Nesta seção serão apresentados dois exemplos de pesquisas que também buscam minimizar dificuldades no uso do QFD, resolvendo uma dificuldade específica do QFD, a definição dos requisitos prioritários dos clientes.

Politis (2003) apresenta os resultados de uma survey realizada nos Emirados Árabes Unidos para entender o efeito de vários níveis e estilos de liderança na aplicação do QFD. A amostra era composta por 14 empresas que utilizavam a série ISO 9000 e o TQM (Total Quality Management - Gestão Pela Qualidade Total). As empresas eram de vários segmentos, incluindo de serviços públicos. Os questionários foram enviados para 145 gerentes de nível médio, tendo um índice de retorno de pouco mais de $70 \%$. Os resultados mostraram que o estilo e tipo de liderança 
afeta a aplicação do QFD e que o apoio da alta administração é crítico para aplicar o método, fornecendo suporte e recursos administrativos para a correta análise dos requisitos dos clientes. O estilo de liderança administrativa "autônoma" tem uma forte relação com o QFD enquanto que o estilo de liderança contigente, com recompensa, tem um efeito negativo no uso do QFD. Segundo Politis (2003), os resultados possibilitam o desenvolvimento de treinamento para os gerentes, e mostra que deve-se buscar um estilo administrativo que envolva participação humana e encoraje a tomada de decisões participativas, ajudando assim apoiar e colaborar com o método do QFD.

Kwong \& Bai (2003) consideram a identificação dos pesos de importâncias dos requisitos dos clientes é essencial para o QFD. O AHP tem sido usado com esta finalidade, mas sua aplicação é mais adequada para tomadas de decisão mais claras, não fuzzy, como são os requisitos dos clientes, que envolvem julgamento humano que na maioria das vezes é impreciso e vago. Kwong \& Bai (2003) propõem então o uso do método fuzzy com o AHP para identificar os pesos dos requisitos mais importantes dos clientes. Segundo os autores, este método se mostrou eficaz devido a sua capacidade de capturar as incertezas do julgamento humano. Entretanto, o método usou números fuzzy triangulares, sendo que esta abordagem não é válida para todas as empresas. Deste modo, será necessário verificar quais são os números fuzzy que devem ser aplicados em cada caso.

\section{Metodologia de pesquisa}

Para Oliveira (1997), as pesquisas podem ser do tipo bibliográfica, documental, de campo, aplicada, teórica, pesquisa de mercado, etc. Esta é uma pesquisa do tipo teórica e tem como objetivo utilizar o projeto axiomático, para desenvolver um modelo de implantação genérico do QFD, que reduza algumas das suas dificuldades de implantação.

O projeto axiomático foi desenvolvido por Suh (1990) com a seguinte lógica: o melhor projeto é aquele que resolver de modo satisfatório o objetivo que o gerou, da forma mais simples possível. Segundo o autor, quanto mais complexo for um projeto menos eficiente ele se torna. Este método envolve a definição do domínio do cliente (requisitos dos clientes) domínio funcional (requisitos funcionais que atendem os clientes) domínio físico (parâmetros de projeto que atendem os requisitos funcionais) e domínio do processo (que atendem os paramentos de projetos) (SUH, 1990). O método está baseado em dois axiomas (axioma da independência e axioma da informação) e de coronárias e teoremas que ajudam a identificar e desenvolver o melhor projeto.

Atualmente, esta pesquisa está na fase de revisão bibliográfica, buscando-se identificar trabalhos sobre dificuldades, pré-requisitos, recomendações sobre o uso do QFD e trabalhos que proponham o uso de outros métodos e ferramentas em conjunto com o QFD, para reduzir suas dificuldades. 
Segundo Lakatos \& Marconi (2002), a pesquisa bibliográfica envolve quatro etapas: Identificação, Localização, Obtenção e Cadastramento das Fontes Bibliográficas. No caso desse estudo, os textos foram localizados utilizando consulta via Internet a sites de bibliotecas e aos periódicos da CAPES. Buscou-se identificar pesquisas que tinham informações sobre dificuldades, pré-requisitos e recomendações para o uso do QFD.

Por enquanto, foram analisados 28 textos sendo que 22 são artigos, uma dissertação de mestrado, uma tese de doutorado e 4 livros. Os dados de interesse dos trabalhos estudados foram digitados numa tabela de quatro colunas criada no excel ${ }^{\circledR}$. A primeira coluna apresenta informações sobre o autor do texto estudado, a segunda informações sobre pré-requisitos da implantação do QFD, a terceira dados sobre as dificuldades de implantação e do uso do método e, finalmente, na quarta coluna foram digitados as recomendações ou métodos apresentados, nas pesquisas estudadas, para diminuir dificuldades específicas do QFD. Os dados desta tabela foram organizados e agrupados em três outras tabelas (Tabela A: Pré-requisitos de implantação; Tabela B: Dificuldades de implantação e no uso do QFD; Tabela C: Recomendações e ferramentas para diminuir dificuldades específicas do QFD) utilizando-se uma adaptação do diagrama de afinidade (método de agrupamento KJ) com o objetivo de facilitar a consulta e análise destes dados. Neste trabalho não será apresentada a metodologia utilizada para o agrupamento dos dados, pois uma descrição detalhada sobre esta etapa foi realizada em um trabalho anterior (CARNEVALLI et al., 2004b). Com o objetivo de verificar quais são os dados já coletados, mais importantes para o uso do QFD em cada uma das três tabelas utilizou-se o método AHP (Analytic Hierarchy Process), apresentado a seguir.

\subsection{Método AHP (Analytic Hierarchy Process)}

Segundo Ohfuji et al. (1997) o procedimento para o uso do método AHP é o seguinte:

1. Montar o formulário do método AHP (na realidade este formulário é uma matriz formada por duas tabelas idênticas);

2. Fazer a comparação de cada item em par, fixando o item da linha e comparando-o com os itens de cada coluna. Para isto, são utilizados os valores comparativos de 1/9 a 9 onde 1- igual importância; 3- pouco mais importante; 5- o item da linha é importante em comparação com o item da coluna; 7- mais importante; 9- muito mais importante; 2, 4, 6 e 8 são números intermediários e os valores $1 / 2$ a $1 / 9$ são usados quando o item da coluna tem um valor superior a 1 em relação ao item fixado na linha;

3. Repetir o procedimento para cada linha, e calcular a média geométrica de cada linha; 
4. Fazer a somatória da coluna da média geométrica e calcular o peso em porcentagem de cada linha dividindo-se o valor da média geométrica com o valor da somatória;

5. Nos casos da tabela da qualidade exigida do QFD, que tem os seus dados agrupados pelo diagrama de afinidades em grupos hierarquizados de $1^{\circ}, 2^{\circ}$ e $3^{\circ}$ níveis, pode-se aplicar o procedimento 1 a 4 para a coluna do $1^{\circ}$ nível e por grupo na coluna do $2^{\circ}$ nível, pertencente em cada item do $1^{\circ}$ nível. Repete-se o processo para o $3^{\circ}$ nível, e neste caso o valor final (grau de importância) dos itens do $3^{\circ}$ nível será dado pela multiplicação dos pesos dos itens de $1^{\circ}, 2^{\circ}$ e $3^{\circ}$ níveis, por agrupamento a que cada item pertença.

\subsubsection{Limitações do método AHP}

Segundo Ohfuji et al. (1997) para se utilizar o método AHP é necessário que o formulário não supere o número máximo de 9 itens na comparação. Nos casos de se trabalhar com tabelas hierarquizadas, como a tabela da qualidade exigida, o número de itens de cada grupo deve ser o mais uniformizado possível, pois sem isto pode ocorrer distorção dos resultados do grau de importância. Outra limitação identificada durante esta aplicação do método AHP é que o seu uso depende do conhecimento que o usuário tem do assunto analisado, sendo assim uma análise subjetiva, que pode ser equivocada.

\subsection{Procedimento adotado}

Como as três tabelas analisadas foram agrupadas pelo método de agrupamento KJ do mesmo modo que seria feito numa tabela de qualidade exigida, buscou-se seguir o mesmo procedimento indicado por Ohfuji et al. (1997), quando se aplica o método AHP para esta tabela do QFD. Entretanto, cada uma das três tabelas analisadas apresentavam um problema, os grupos de cada nível não eram uniformes, o que significava que os graus de importância calculados nos últimos itens não poderiam ser usados por serem um valor distorcido. Deste modo, os valores úteis para uma análise desta tabela são os pesos de cada nível hierárquico (coluna da tabela ou de cada grupo destes níveis).

Outro problema encontrado ao se aplicar o método AHP foi a existência de alguns itens isolados em cada nível hierarquizado, quando considerados por grupos. Este fato ocorreu porque ao se aplicar o método KJ buscou-se evitar, quando possível, a criação de itens (títulos que representassem o grupo, ou desdobramento de um item mais abstrato em mais concreto, que é realizado pelo pesquisador, chamado neste trabalho de proponente, ao aplicar o método KJ), pois só serão utilizados na aplicação do projeto axiomático os itens com referência na literatura. Nestes 
casos, ao se aplicar o AHP, estes itens não seriam comparados com ninguém, ficando com peso igual a 1. Uma solução adotada, para os casos em que o número de itens da coluna não superasse os 9 itens, era comparar todos os itens da coluna juntos e não em grupos, como se eles fossem do nível 1, seguindo os procedimentos de 1 a 4 de Ohfuji et al. (1997). Nas colunas que o número de itens superasse 9 itens foi seguido o procedimento do item 5. Deste modo, foi possível fazer a análise de grau de importância pelos pesos de cada item separadamente por coluna, e nos casos das colunas com mais de 9, fez-se a análise por grupo, este fato não prejudica o método, já que o grau de importância pela multiplicação dos pesos não foi utilizado, por terem seus valores distorcidos pela falta de uniformidade de cada tabela. Na Tabela 1 é apresentado um exemplo de uma matriz AHP.

\begin{tabular}{|c|c|c|c|c|c|}
\hline $2{ }^{\circ}$ Nível & $\begin{array}{l}\text { Pressupostos Implícitos do } \\
\text { QFD: "Formalidade e } \\
\text { hierarquia (Guimarães, p. 92; } \\
\text { 2003)" Para usar o QFD a } \\
\text { empresa deve ter um certo } \\
\text { grau de organização e } \\
\text { comando (GUIMARÃES, } \\
\text { 2003) } \\
\end{array}$ & $\begin{array}{l}\text { Desenvolver o conhecimento } \\
\text { necessário para implementar o } \\
\text { QFD. Se não tem este } \\
\text { conhecimento, deve-se buscar o } \\
\text { apoio necessário para ajudar no } \\
\text { treinamento e na implantação. } \\
\text { (CARNEVALLI \& MIGUEL, } \\
\text { 2003) }\end{array}$ & $\begin{array}{c}\text { Avaliar a } \\
\text { implantação } \\
\text { (Definido } \\
\text { pelo autor - } \\
\text { proponente) }\end{array}$ & $\begin{array}{c}\text { Média } \\
\text { Geométrica }\end{array}$ & PESO \\
\hline $\begin{array}{l}\text { Pressupostos Implícitos do } \\
\text { QFD: "Formalidade e } \\
\text { hierarquia (Guimarães, p. } \\
\text { 92; 2003)" Para usar o } \\
\text { QFD a empresa deve ter } \\
\text { um certo grau de } \\
\text { organização e comando } \\
\text { (GUIMARÃES, 2003) }\end{array}$ & 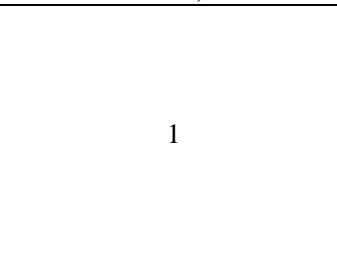 & ( & 3 & 0,75 & 0,16 \\
\hline $\begin{array}{c}\text { Desenvolver o } \\
\text { conhecimento necessário } \\
\text { para implementar o QFD. } \\
\text { Se não tem este } \\
\text { conhecimento, deve-se } \\
\text { buscar o apoio necessário } \\
\text { para ajudar no treinamento } \\
\text { e na implantação. } \\
\text { (CARNEVALLI \& } \\
\text { MIGUEL, 2003) }\end{array}$ & 7 & 1 & 7 & 3,66 & 0,77 \\
\hline $\begin{array}{l}\text { Avaliar a implantação } \\
\text { (Definido pelo autor - } \\
\text { proponente) }\end{array}$ & $1 / 3$ & $1 / 7$ & 1 & 0,36 & 0,08 \\
\hline & & & Total & 4,78 & 1 \\
\hline
\end{tabular}

Tabela 1 - Aplicação do AHP na segunda coluna da tabela A.

\section{Análise dos resultados}

Será apresentada apenas a análise parcial dos principais resultados de cada uma das três tabelas (A, B e C), que estão sendo desenvolvidas, pois estas tabelas são muito grandes não permitido a apresentação de todas as suas colunas, neste artigo. Optou-se então por apresentar apenas algumas colunas (como exemplo) e os principais resultados das demais.

\subsection{Análise da tabela A: Pré-requisitos de implantação do QFD}

$\mathrm{Na}$ análise da tabela A verifica-se que na primeira coluna existe apenas um item, relacionado com "a necessidade de apoio da alta administração, tanto em liderança como na liberação de recursos, para se implementar o QFD” (com base em CARNEVALLI \& MIGUEL, 2003; CHENG 
et al., 1995; CRISTIANO et al., 2001; GOVERS, 1996; GRIFFIN, 1992; KATHAWALA \& MOTWANI, 1994; OHFUJI et al., 1997). Este fato ocorre devido ao uso do método KJ para organizar os dados de cada tabela. Com este método os dados são organizados de forma hierarquizada do nível mais abstrato ( $1^{\circ}$ nível) ao mais concreto. Assim, verifica-se que o apoio da alta administração além de ser o item mais abstrato, também interfere indiretamente ou diretamente com os demais itens de pré-requisitos de implantação do QFD. Os demais itens, de certo modo, podem ser considerados, como um detalhamento deste item. Desta maneira, o apoio da alta administração é o item mais importante da tabela A.

Analisando-se a $2^{\text {a }}$ coluna da tabela A apresentada na tabela 1 , verifica-se que o item de maior peso está relacionado com "o desenvolvimento do know how necessário para implementar o QFD”, com peso 0,77. Deste modo, a empresa não deve subestimar os recursos necessários para o treinamento e implantação do QFD.

$\mathrm{Na}$ análise da $3^{\mathrm{a}}$ coluna ( $3^{\circ}$ nível) da tabela $\mathrm{A}$, apresentada na tabela 2 , novamente se destaca o item relacionado com "o treinamento do QFD”, com peso de 0,40 (40\%). Neste caso, sendo este um item mais concreto que o da $2^{\text {a }}$ coluna, a literatura estudada (GOVERS, 1996; GUIMARÃES, 2003; KATHAWALA \& MOTWANI, 1994) recomenda que o treinamento em QFD envolva além das equipes que usam o método também as pessoas que com ele terão contato mesmo de forma não direta (por exemplo: gerentes de departamentos).

\begin{tabular}{|l|c|}
\hline $3^{\circ}$ Nível & PESO \\
\hline $\begin{array}{l}\text { Para usar o QFD existe a necessidade de um excelente gerenciamento do trabalho do dia a dia das } \\
\text { funções envolvidas com o QFD (CHENG et al., 1995) }\end{array}$ & 0,13 \\
\hline $\begin{array}{l}\text { Realizar o treinamento necessário de todos os envolvidos para eliminar paradigmas que possam } \\
\text { prejudicar o projeto (GOVERS, 1996; GUIMARÃES, 2003; KATHAWALA \& MOTWANI, } \\
\text { 1994) }\end{array}$ & 0,40 \\
\hline $\begin{array}{l}\text { Armazenar e utilizar informações da empresa na rotina diária. O QD realiza esta função. } \\
\text { (OHFUJI, ONO \& AKAO; 1997). }\end{array}$ & 0,06 \\
\hline Fazer desdobramentos dos custos (Definido pelo autor - proponente) & 0,18 \\
\hline $\begin{array}{l}\text { Pressupostos Implícitos do QFD: A equipe deve ter um tempo policrônico e autonomia para o uso } \\
\text { do QFD, ou seja, nos projetos não existem um cronograma e pontos feedback rígidos } \\
\text { (GUIMARÃES, 2003) }\end{array}$ & 0,03 \\
\hline $\begin{array}{l}\text { Deve-se fazer a avaliação da implantação do QFD e registrar as melhorias alcançadas, para } \\
\text { verificar a eficácia no uso do método. (CARNEVALLI \& MIGUEL, 2003) }\end{array}$ & 0,19 \\
\hline Total & 1,00 \\
\hline
\end{tabular}

Tabela 2 - Resultado da aplicação do AHP na terceira coluna da tabela A.

A $4^{\mathrm{a}}$ coluna da tabela $\mathrm{A}$, apresentada na tabela 3 mostra que o item que mais se destacou (peso 0,36) está relacionado com "manter a fidelidade da informação durante todo o processo" (GUIMARÃES, 2003), ou seja, identificar os requisitos mais importantes para os clientes e conseguir atendê-los no produto final sem distorce-los no processo. Para isto a recomendação dada por Guimarães (2003) é "manter a comunicação com os clientes internos e externos durante todo o desenvolvimento do projeto". 
Outro item que se destaca nesta coluna, dada por Guimarães (2003), é o "trabalho em equipe” que teve peso 0,23. Segundo Miguel (2001), o trabalho em equipe é uma maneira de realizar trocar experiências entre seus membros de modo que cada decisão seja o resultado de um consenso.

\begin{tabular}{|c|c|}
\hline $4^{\circ}$ Nível & PESO \\
\hline $\begin{array}{l}\text { Pressuposto Implícito do QFD: As reuniões da equipe do QFD devem ser bem preparadas e ter } \\
\text { um certo nível de disciplinas durante a sua execução (GUIMARÃES, 2003) }\end{array}$ & 0,02 \\
\hline Pressuposto Implícito do QFD: Trabalho em equipe (GUIMARÃES, 2003) & 0,23 \\
\hline Coletar dados primitivos (Definido pelo autor - proponente) & 0,15 \\
\hline $\begin{array}{l}\text { Pressuposto Implícito do QFD: Para manter a fidelidade das informações dos clientes no } \\
\text { processo do QFD, deve-se manter o contato com os clientes internos e externos até a conclusão } \\
\text { do projeto (GUIMARÃES, 2003). }\end{array}$ & 0,36 \\
\hline Experiência em engenharia de valor (KATHAWALA \& MOTWANI, 1994) & 0,02 \\
\hline Realizar a Matriz da Qualidade para arquivar informações (OHFUJI, ONO \& AKAO; 1997) & 0,04 \\
\hline $\begin{array}{l}\text { Deve-se registrar o conhecimento dos projetos num manual, para orientar futuras aplicações do } \\
\text { QFD.(CARNEVALLI \& MIGUEL, 2003) }\end{array}$ & 0,16 \\
\hline Total & 1,00 \\
\hline
\end{tabular}

Tabela 3 - Resultado da aplicação do AHP na quarta coluna da tabela A.

$\mathrm{Na} 5^{\mathrm{a}}$ coluna os itens que mais se destacaram estão relacionados com:

1. “Características da equipe para usar o QFD” (definido pelo proponente), com peso 0,24. Estas características estão relacionadas com a competência, conhecimento técnico, departamento pertencente, facilidade em se trabalhar em grupo, além de nível de motivação e comprometimento de cada membro da equipe;

2. "Nível de envolvimento e colaboração dos departamentos e nível de comunicação com os clientes", com peso 0,21. Segundo Guimarães (2003) que definiu este item, quanto maior a participação e colaboração dos departamentos e quanto mais a equipe se comunica com os clientes, melhor será o rendimento do projeto. Isto porque o rendimento do projeto melhoraria se o aumento da comunicação com os clientes facilitasse a identificação da qualidade exigida, ao eliminar dúvidas sobre os seus requisitos. Além disso, uma maior participação e contribuição dos departamentos aumentariam a troca de conhecimento e o apoio ao projeto.

As colunas $6^{\mathrm{a}}$ e $7^{\mathrm{a}}$ da tabela $\mathrm{A}$, foram analisadas por grupo por terem mais de 9 itens por coluna, seguindo o procedimento 5 apresentado no tópico 3.1. Estes itens não serão apresentados neste trabalho, pois podem ser considerados como um detalhamento da $5^{\text {a }}$ coluna da mesma tabela e não acrescentaria valor a presente análise.

\subsection{Análise da tabela B: Dificuldades de implantação e no uso do QFD}

Analisando-se os itens da tabela B com o método AHP, verificou-se que na $1^{\text {a }}$ coluna existe apenas um item, dificuldades por "falta de recursos" (CARNEVALLI \& MIGUEL, 2003). Isto 
significa que as demais dificuldades de implantação do QFD podem ser influenciadas direta ou indiretamente por esta dificuldade, pois é do $1^{\circ}$ nível de abstração.

$\mathrm{Na} 2^{\mathrm{a}}$ coluna da tabela $\mathrm{B}$, apresentada na tabela 4 , as dificuldades que mais se destacaram estão relacionadas com "dificuldade de transferir as informações do desenvolvimento para a produção" (CHENG et al., 1995), com peso 0,41. Isto porque muitos dados foram gerados no uso do método e é necessário apresenta-los de forma organizada, clara e definidas as responsabilidades, para conscientizar a produção sobre a importância de se atender estas especificações para os clientes. Também destaca-se na $2^{\mathrm{a}}$ coluna as "dificuldades por falta de conhecimento em QFD" (CARNEVALLI \& MIGUEL, 2003) com peso 0,36. Este item mostra a coerência dos dados da tabela B como os da tabela $\mathrm{A}$ (pré-requisitos), pois se a empresa não atender o pré-requisito treinamento pode gerar dificuldades na implantação do QFD, por falta de conhecimento no método.

\begin{tabular}{|l|c|}
\hline $2^{\circ}$ Nível & PESO \\
\hline Dificuldade por falta de conhecimento no QFD (CARNEVALLI \& MIGUEL, 2003) & 0,36 \\
\hline Dificuldade com a equipe do QFD (Definido pelo autor - proponente) & 0,15 \\
\hline Dificuldade de avaliar o sucesso do projeto do QFD (Definido pelo autor - proponente) & 0,07 \\
\hline $\begin{array}{l}\text { Dificuldade de transferir as informações do desenvolvimento para a produção (CHENG et al, } \\
\text { 1995) }\end{array}$ & 0,41 \\
\hline Total & 1,00 \\
\hline
\end{tabular}

Tabela 4 - Resultado da aplicação do AHP na segunda coluna da tabela B.

$\mathrm{Na} 3^{\mathrm{a}}$ coluna da tabela B ( $3^{\circ}$ nível), apresentada na tabela 5 , se destacou, com peso 0,48 , a dificuldade relacionada com "falta de comprometimento da equipe do QFD" (CARNEVALLI \& MIGUEL, 2003; GRIFFIN, 1992). Este problema também já foi apresentado na tabela A, sobre os pré-requisitos, mostrando novamente a coerência entre as duas tabelas.

\begin{tabular}{|l|c|}
\hline $3^{\text {}}$ Nível & PESO \\
\hline Dificuldade de como fazer e preencher as tabelas e matrizes (CHENG, 2003) & 0,16 \\
\hline $\begin{array}{l}\text { Dificuldade por falta de comprometimento da equipe do QFD (CARNEVALLI \& MIGUEL, } \\
\text { 2003; GRIFFIN, 1992) }\end{array}$ & 0,48 \\
\hline $\begin{array}{l}\text { Dificuldade pelos benefícios tangíveis só aparecerem a longo prazo (GRIFFIN, 1991; GRIFFIN } \\
\text { 1992) }\end{array}$ & 0,08 \\
\hline $\begin{array}{l}\text { Dificuldade pelo o sucesso do projeto ser avaliado de forma subjetiva pelos participantes } \\
\text { (CHENG, 2003) }\end{array}$ & 0,03 \\
\hline Dificuldade de fazer o QFDr (CHENG, 2003) & 0,24 \\
\hline Total & 1,00 \\
\hline
\end{tabular}

Tabela 5 - Resultado da aplicação do AHP na terceira coluna da tabela B.

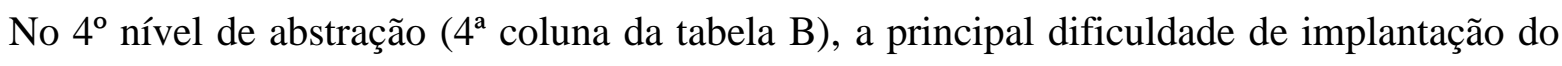
método está relacionado com "fazer pesquisa eficiente de mercado" (segundo o proponente), com peso 0,32 . Neste item, as dificuldades se referem a falta de recursos, além de como definir a melhor amostra e técnica de coleta de dados para a pesquisa. Outra dificuldade que se destaca neste item é realizar o modelo do QFD japonês com seus 4 desdobramentos, num projeto piloto (AKAO, 1996), o que não é recomendado pelo próprio Akao, devido a falta de experiência da empresa no uso do método. 
$\mathrm{Na} 5^{\mathrm{a}}$ coluna, as dificuldades que se destacaram estão relacionadas com "resolver gargalos de engenharia e problemas de custos" (segundo o proponente), cada uma com peso 0,18. Também nesta coluna se destaca uma dificuldade muito citada na literatura (DEAN, 1992; VASCONCELOS et al., 2003; WASSERMAN, 1993), "a dificuldade de se definir a qualidade projetada". Neste caso, o valor dado a características da qualidade vai depender da sua importância para satisfazer os requisitos dos clientes, a interação que cada item das características da qualidade tem entre si, a análise competitiva e do plano estratégico da empresa (objetivos a serem atingidos pelo projeto).

As $6^{\mathrm{a}}$ e $8^{\mathrm{a}}$ colunas da tabela B foram analisadas em grupo, pois elas têm mais de 9 itens cada uma. Neste trabalho também não serão apresentados os resultados destas colunas, pois podemos considerá-las como sendo um detalhamento dos itens da $5^{\text {a }}$ coluna da mesma tabela.

\subsection{Análise da tabela $\mathrm{C}$ : Recomendações e ferramentas para diminuir dificuldades específicas do QFD}

$\mathrm{Na}$ análise dos 9 itens pertencentes a $1^{\mathrm{a}}$ coluna da tabela $\mathrm{C}$, utilizando-se o método AHP, o item que mais se destacou, esteve relacionado com "verificar a satisfação dos clientes" (definido pelo proponente), que teve peso 0,29. Esta é uma recomendação importante pois ajuda a verificar a eficácia ou não da aplicação do QFD. Em segundo lugar se destacou "a elaboração do PTP" (definido pelo proponente), com peso de 0,18 , pois é uma das tabelas importantes, para transferir de modo eficiente e eficaz os resultados das matrizes do QFD para a manufatura, resolvendo a dificuldade apresentada na segunda coluna da tabela B. Em terceiro, se destacou o item sobre a “consolidação do QFD” (também definido pelo proponente), com peso 0,13. Este item também era esperado como importante, pois somente com a consolidação do método é que a empresa consegue atingir todos os seus benefícios.

Como a $2^{\mathrm{a}}$ coluna da tabela $\mathrm{C}$ tem mais de nove itens, o método AHP foi aplicado separadamente em cada um dos 9 grupos pertencentes a esta coluna, conforme o procedimento 5 apresentado no tópico 3.1. Os itens que se destacaram nesta coluna foram:

1. “A recomendação da realização da análise do PDS (Sistema de Desenvolvimento de Produto)"da empresa previsto por CHENG (2003), esta análise é importante para verificar se a empresa precisa ou não modificar o seu PDS e se deve ou não utilizar o QFD para esta finalidade;

2. Mais uma vez "o treinamento em QFD” (definido pelo proponente) aparece como uma recomendação importante, podendo resolver a dificuldade por "falta de conhecimento em QFD" apresentada na tabela B. Esta recomendação é detalhada na terceira coluna, "recomendando o uso de módulos de treinamento " (GUIMARÃES, 2003), “o uso do Modus Operandi: Aprender, 
Aplicar, Ensinar e Melhorar" para difundir o QFD (CHENG et al., 1995); "usar as aplicações anteriores do QFD (já concluídas na empresa), para o treinamento" (CRISTIANO et al. 2001). A tabela vai detalhando o assunto até a $5^{\mathrm{a}}$ coluna, onde os itens que mais se destacam são "realizar treinamento de 6 horas para o pessoal envolvido e de uma semana para a equipe que usa o QFD” (CHENG et al., 1995);

3. A "recomendação de se realizar a matriz da qualidade", sugerida pelo proponente, se destacou como importante no maior grupo da $2^{\mathrm{a}}$ coluna, com 9 itens. Isto porque a matriz da qualidade é uma das mais importantes do QFD, por identificar os requisitos prioritários dos clientes, traduzidos em especificações de projetos, além de fazer análise competitiva e definir os valores objetivos de cada especificação. Por esta razão AKAO (1996) também recomenda o seu uso, mesmo nos casos em que os clientes fornecem as especificações do projeto;

4. Segundo CHENG (2003), para garantir a qualidade é necessário "elaborar e executar o modelo conceitual e o PTP (Padrão Técnico de Processo)”, com peso 0,46. Isto porque, o desenvolvimento apenas da matriz da qualidade não é suficiente, para a empresa conseguir fabricar produtos com as especificações de projeto definidas nesta matriz. É necessário também fazer outros desdobramentos até o processo de fabricação (definido pelo modelo conceitual) e transferir as informações resultantes do QFD numa tabela que o operador entenda e verifique as suas responsabilidades (PTP).

5. Em relação a consolidação do método do QFD, o que se destacou foi a "necessidade de ampliação da infraestrutura de desenvolvimento do produto" (CHENG et al., 1995);

6. No $8^{\circ}$ grupo ocorreu um detalhamento do $7^{\circ}$ item da $1^{\mathrm{a}}$ coluna, sobre a verificação da satisfação dos clientes, com as seguintes recomendações: "uso de amostragem, e de planejamento de experimentos, além de testes sensoriais, entre outros" (CHENG et al., 1995).

Como os demais itens da tabela $\mathrm{C}$ podem ser considerados um desdobramento da $2^{\mathrm{a}}$ coluna só serão apresentados neste artigo os itens diretamente relacionados com as principais dificuldades no uso do QFD.

Para resolver "os problemas de falta de comprometimento dos membros da equipe" apresentados na tabela $\mathrm{B}$, a tabela $\mathrm{C}$ recomenda, na $4^{\mathrm{a}}$ coluna, cinco possibilidades sendo que as três que se destacam são:

1. "Os gerentes participarem das reuniões para quebrar as dificuldades da equipe" (GRIFFIN, 1992), com peso de 0,35 ;

2. "Ter um enfoque de tomada de decisão em grupo, motivando a participação e colaboração entre os membros da equipe" (GUIMARÃES, 2003), com peso 0,28;

3. "Escolher para a equipe pessoas que já tenham um entrosamento entre si" (GRIFFIN, 1992), com peso 0,22 . 
Para resolver "a dificuldades de se fazer pesquisa eficaz de mercado" (na tabela B) a tabela $\mathrm{C}$ na $5^{\mathrm{a}}$ coluna recomenda "o uso do questionário do modelo de Kano" (MATZLER \& HINTERHUHER, 1998), com peso 0,67.

Sobre "a dificuldade de se fazer o modelo do QFD com quatro desdobramentos, num projeto piloto", Akao (1996) recomenda na tabela C, na $4^{\text {a }}$ coluna, iniciar o projeto piloto só com o desdobramento da qualidade, acrescentando mais o desdobramento da tecnologia no $2^{\circ}$ projeto, o desdobramento dos custos no $3^{\circ}$ e só no $4^{\circ}$ projeto realizar os 4 desdobramentos.

Sobre "como resolver gargalos de engenharia" existem três possibilidades na $4^{\text {a }}$ coluna (todas definidas pelo proponente) que são:

1. "Investir na resolução do gargalo", a qual é detalhada na $5^{\text {a }}$ coluna com "o uso de Benchmarking", com peso 0,60; "investir no desenvolvimento da tecnologia necessária", com peso 0,20, “comprar a tecnologia", também com peso 0,20 (CHENG et al., 1995).

2. "Terceirizar a resolução do problema" que é detalhado por CHENG et al. (1995) como terceirizar para universidades ou centros de pesquisas.

3. "Usar método direto para resolver o problema, como PDCA; Planejamento de Experimento; etc." (CHENG et al., 1995).

Para resolver os problemas de custos o item que mais se destaca na tabela $\mathrm{C}$ é "realizar o desdobramento dos custos tradicional do QFD” (CHENG et al., 1995).

Sobre as dificuldades de definir a qualidade projetada, a tabela C apresenta várias recomendações e as que mais se destacam, são "o uso do método de Taguchi”" (na $7^{\text {a }}$ coluna) com peso 0,35 (MAIER, 1995); Fazer a "matriz características da qualidade versus características da qualidade" (CHENG et al., 1995) para se verificar as interações entre si (6 $6^{\mathrm{a}}$ coluna, peso 0,71) e "fazer análise competitiva" (também na 6 coluna), com peso 0,69 (CHENG et al., 1995). A seguir é apresentada a conclusões deste trabalho.

\section{Conclusões}

O uso do método AHP se mostrou eficaz para a priorização dos itens já estudados, verificando, nos casos das colunas com menos de 9 itens, quais são os pré-requisitos do QFD que são prioritários, quais as principais dificuldades de implantação e quais as melhores recomendações, ou ferramentas menos complicadas de utilizar junto com o QFD, para resolver suas dificuldades. Nos casos de colunas com mais de 9 itens, o uso do método AHP ocorreu apenas por agrupamento. Como as tabelas não estavam com os números de cada item organizados uniformemente não foi possível calcular, de forma confiável, o grau de importância para os itens de maior nível, mas pode- 
se utilizar a análise pelos pesos de cada item. Deste modo, esta foi uma limitação do método. Outra limitação do método é ser uma análise subjetiva realizada pelo pesquisado que o aplica, podendo ser equivocada.

Com a análise da tabela A (pré-requisitos de implantação do QFD) verificou-se que o apoio da alta administração, desenvolve o know how em QFD com todos os envolvidos, além de trabalho em equipe, e características dos seus membros são pré-requisitos importantes para a implantação. Também nesta tabela são apresentados alguns cuidados que devem ser tomados na implantação, como manter a fidelidade nos desdobramentos dos requisitos dos clientes.

Com a análise da tabela B (dificuldades de implantação e no uso do QFD) verificou-se que as dificuldades mais importantes a serem resolvidas estão relacionadas com: falta de recursos e treinamento, dificuldade de se fazer pesquisa de mercado; falta de comprometimento da equipe; dificuldades de transferir as informações para a fabricação e de se resolver problemas de custos e de gargalos de engenharia.

$\mathrm{Na}$ análise da tabela $\mathrm{C}$ (recomendações e ferramentas para diminuir dificuldades específicas do QFD) os itens de $1^{\circ}$ e $2^{\circ}$ níveis que mais se destacam estiveram relacionados com recomendações para medir a eficácia do QFD pela satisfação dos clientes, utilizar o PTP para transferir as informações do QFD de modo eficiente e eficaz para a produção, e verificar a necessidade de se melhorar a estrutura da empresa para se consolidar o QFD. Também se verificou nas demais colunas quais eram as recomendações para resolver os principais problemas identificados na tabela B.

Posteriormente a este estudo foi a elaboração da tabela D, onde se verificou, com base na literatura, quais são os principais motivos que levaram as empresas a utilizar o método do QFD, sendo que estes dados também foram organizados pelo método de agrupamento KJ (sem adaptações) e se utilizou o método AHP, para identificar os itens prioritários. Com a análise dos dados priorizados pelo AHP, verificou-se que a maioria dos motivos para se aplicar o QFD está diretamente relacionado com a busca da melhoria da capacidade competitiva da empresa no seu mercado de atuação.

A próxima etapa desta pesquisa envolve conclusão de cada uma das 4 tabelas, estudar os dados coletados na presente pesquisa bibliográfica e a realização de uma pesquisa de campo com empresas que utilizam o QFD para ajudar a definir o domínio do Cliente e iniciar a aplicação do $\mathrm{AD}$, para atender os objetivos mais abrangentes desse projeto de pesquisa.

\section{Agradecimentos}

Os autores agradecem o apoio recebido pela CAPES (bolsa de doutorado). 


\begin{abstract}
QFD is an important method for product development. The method translates and identifies customer s' requirements into measurable product characteristics and deploys these characteristics for each stage of a product or service development conducted. However, the bibliography shows various difficulties of QFD application. A literature review is realizing to identify difficulties, requirement and recommendation of QFD usage. This paper shows the use of AHP (Analytic Hierarchy Process) to identify those main difficulties. In the future this research will use this result to develop procedure that aims at reducing some of those difficulties.
\end{abstract}

Key words: QFD; AHP; Difficulties of QFD use.

\title{
5. Referências
}

AKAO, Y. Introdução ao desdobramento da qualidade. Belo Horizonte : Fundação Christiano Ottoni. 1996.

AKAO, Y.; MAZUR, G. H. The leading edge in QFD: past, present and future. International Journal of Quality \& Reliability Management, v. 20, n. 1, p. 20-35, 2003.

\section{cross ${ }^{\text {ref }}$}

BOUCHEREAU; V.; ROWLANDS,H. Methods and techniques to help quality function deployment (QFD). Benchmarking: An International Journal, v. 7, n. 1, p. 8-16, 2000.

CARNEVALLI, J. A. Estudo exploratório tipo survey sobre o uso do QFD nas 500 maiores empresas no Brasil. 2002. 148 f. Dissertação (Mestrado em Engenharia de Produção) - Programa de Pós-Graduação em Engenharia de Produção - UNIMEP. 2002.

CARneVAlli, J. A.; MIGUEL, P. A. C. Empresas de Referência na Utilização do Desdobramento da Função Qualidade. In: CONGRESSO BRASILEIRO GESTÃO DE DESENVOLVIMENTO DE PRODUTO, 4., 2003, Gramado. Anais...Gramado, 2003. [CD-ROM].

CARNEVALLI, J. A. et al. Análise de dados da literatura sobre a implantação do QFD utilizando o método AHP. In: SIMPÓSIO DE ENGENHARIA DE PRODUÇÃO, 11., 2004, Bauru. Anais... Bauru, 2004a. Disponível em: <http://www.simpep.feb.unesp.br/anteriores.html.>. Acesso em: 6 maio 2003.

CARNEVALLI, J. A. et al. Organização de dados da literatura sobre os requisitos, dificuldades e recomendações do uso do QFD utilizando uma adaptação do diagrama de afinidade. In: SIMPÓSIO DE ENGENHARIA DE PRODUÇÃO, 11., 2004, Bauru. Anais... Bauru, 2004b. Disponível em: 〈http://www. simpep.feb.unesp.br/anteriores.html.>. Acesso em: 6 maio 2003.

CHAN, L. K.; WU, M. L.Quality function deployment: A literature review. European Journal of Operational Research, v. 143, n.3, p. 463-497, 2002.

cross ref

CHENG, L. C. et al. QFD: planejamento da qualidade. Belo Horizonte : Fundação Christiano Ottoni, 1995.

CHENG, L. C. QFD in product development: methodological characteristics and a guide for intervention. nternational Journal of Quality \& Reliability Management, v. 20, n. 1, p. 107-122, 2003.

CRISTIANO, J. J. et al. Key factors in the successful application of quality function deployment (QFD). IEEE Transactions on Engineering Management, v. 48, n. 1, p. 81-95, 2001.

cross ref

DEAN, E. B. Quality function deployment for large systems. In: INTERNATIONAL ENGINEERING MANAGEMENT CONFERENCE, 1992, Eatontown. Proceedings... Eatontown. Oct. 1992. p. 25-28.

GOVERS, C. P. M. What and how about quality function deployment (qfd). International Journal of Production Economics, v. 46-47, p. 575-585, Dec. 1996. 
GRIFFIN, A. Evaluating development processes: QFD as an example. Cambridge, Massachusetts, Marketing Science Institute, 1991. Disponível em: <http://www.msi.org/msi/meetings.cfm>. Acesso em: 7 ago Eatontown. 2003. Report Summary 91-121.

GRIFFIN, A. Evaluating QFD's use in US firms as a process for developing products. Journal of Product Innovation Management, v. 9, n. 3, p.171-187, Sept. 1992.

cross ref

GUIMARÃES, L. M. QFD - Quality function deployment: uma análise de aspectos culturais organizacionais como base para definição de fatores críticos de sucesso (FCS) na implementação da metodologia. 2003. Dissertação (mestrado profissional) - Universidade Estadual de Campinas. 2003.

KATHAWALA, Y.; MOTWANI, J. Implementing quality function deployment. Research and Concepts, v. 6, n. 6, p. 31-35, 1994.

KWONG, C. K.; BAI, H. Determining the importance weights for the customer requirements in qfd using a fuzzy ahp with an extent analysis approach. IIE Transactions, v. 35, n. 7, p. 619-626, 2003.

cross ref

LAKATOS, E. M.; MARCONI, M. D. A. Técnicas de pesquisa. 5. ed. São Paulo : Atlas, 2002.

MARTINS, A.; ASPINWALL, E. M. Quality function deployment: an empirical study in the UK. Total Quality Management, v. 12, n. 5, p. 575-588, 2001.

crossef

MIGUEL, P. A. C. Qualidade: enfoques e ferramentas. São Paulo : Artliber, 2001.

MATZLER, K.; HINTERHUBER, H. H. How to make product development projects more successful by integrating Kano's model of customer satisfaction into quality function deployment. Technovation, v. 18, n. 1, p. 2538,1998 .

cross ref

MIGUEL, P. A. C. The state-of-the-art of the Brazilian QFD applications at the top 500 companies. International Journal of Quality \& Reliability Management, v. 20, n. 1, p. 74-89, 2003.

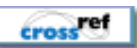

PARKIN, N. et al. The introduction of QFD in a UK original equipment manufacturer. Managerial Auditing Journal, v. 17 , n. 1 , p. 43-54, 2002.

cross ref

POLITIS, J. D. QFD: the role of various leadership styles. Leadership \& Organization Development Journal, v. 24, n. 4, p. 181-192, 2003.

cross ref

OHFUJI, T. et al. Métodos de desdobramento da qualidade (1). Belo Horizonte : Fundação Christiano Ottoni, 1997.

OLIVEIRA, S. L. Tratado de metodologia científica: projetos de pesquisas, TGI, TCC, monografias, dissertações e teses. São Paulo : Pioneira, 1997.

SUH, N. P. The principles of design. New York : Oxford University Press, 1990.

VASCONCELOS, E. C. et al. Determinação de valores objetivos em matrizes QFD usando delineamento de experimentos. In: ENCONTRO NACIONAL DE ENGENHARIA DE PRODUÇÃO, 23., 2003, Ouro Preto. Anais ... São Paulo: NovoDisc Brasil Indústria Fonográfica Ltda., 2003. [CD-ROM].

WASSERMAN, G. S. On how to prioritize design requirements during the QFD planning process. IIE Transactions, v. 25, n. 3, p. 59-65, 1993. 